\title{
ÉVOLUTION HISTORIQUE DU TRAVAIL SOCIAL EN TURQUIE: DE LA RÉPUBLIQUE À NOS JOURS
}

\author{
Bekir GÜZEL ${ }^{a}$
}

\begin{abstract}
Résume
Dans cette étude, les pratiques de travail social menées en Turquie après 1923 seront systématiquement discutées dans une perspective chronologique. Bien que le travail social ait commencé comme une profession en Turquie dans les années 1960, diverses activités pour les services sociaux ont été menées immédiatement après la proclamation de la république. Ces activités ont constitué une base importante pour que le travail social devienne une profession en Turquie. À la suite des activités mondiales des Nations Unies, en particulier après la Seconde Guerre mondiale, les études et les efforts qui ont commencé en Turquie ont contribué à la formation et à la professionnalisation du travail social. Les politiques néolibérales qui se sont répandues dans le monde dans les années 1980 ont également affecté le travail social en Turquie. Cependant, on peut affirmer que le changement le plus radical et le plus vaste a eu lieu après le millénaire. Dans cette étude, ces périodes sont discutées en détail. Il y a deux points importants qui distinguent cette étude des études précédentes. Le premier de ces points est que cette étude est traitée dans une structure chronologique différente et discutée avec une perspective critique. Un autre point est que cette étude est rédigée en français, contrairement aux langues précédemment publiées (turc, anglais ou allemand).
\end{abstract}

Mots-clés: Turquie, proclamation de la république, travail social, Nations Unies, institutionnalisation.

$$
\text { 邹淡 }
$$

\section{HISTORICAL EVOLUTION OF SOCIAL WORK IN TURKEY: FROM THE REPUBLIC TO THE PRESENT DAY}

\section{Abstract}

In this study, social work practices carried out in Turkey after 1923 is discussed from a chronological perspective. Although social work started as a profession in Turkey in the 1960s, various activities for social services were carried out immediately after the proclamation of the republic. These activities were an important basis for social work to become a profession in Turkey. As a result of the global activities of the United Nations especially after the Second World War, the studies and efforts that started in Turkey

a Dr. Öğr. Üyesi, Recep Tayyip Erdoğan Üniversitesi, Sağlık Bilimleri Fakültesi, Sosyal Hizmet Bölümü, bekir.guzel@erdogan.edu.tr

Makale Geliş Tarihi: 27.06.2021, Makale Kabul Tarihi: 07.09.2021 
contributed to full-time social work education and professionalization. The neoliberal policies that spread to the world in the 1980s also affected social work in Turkey. However, it can be stated that the most radical and wide change took place after the millennium. In this study, these periods are discussed in detail. There are two important points that distinguish this study from previous studies. The first of these points is that this study is handled in a different chronological structure and discussed with a critical perspective. Another point is that this study is written in French, unlike the previously published languages (Turkish, English or German). Keywords: Turkey, proclamation of the republic, social work, United Nations, institutionalization.

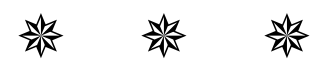

\section{Introduction}

L'historicité des activités philanthropiques et de services sociaux en Anatolie a commencé avec l'État seldjoukide. Les activités philanthropiques sont restées des fondations sociales en Anatolie à travers l'Empire ottoman et ont permis de continuer à fournir des services sociaux. Cependant, l'existence et la fonction des fondations maintenaient l'empire à l'arrière-plan des études publiques et institutionnelles des services sociaux (Ertem, 1999). Pour cette raison, il a fallu des siècles pour institutionnaliser et faire connaître le travail social dans la Région anatolienne. Dans la dernière période de l'Empire ottoman, en particulier après le Hatt-i Sharif de Gulhane (l'Édit du Tanzimat), les efforts de réforme ont déplacé l'accent mis sur les politiques et sociales vers les services sociaux. Au cours de cette période, des règlements et des lois ont été adoptés pour les travailleurs travaillant dans les mines. Des institutions ont également été développées pour protéger les enfants, les femmes et les pauvres sans abri comme Dulhâne (1884), Darüşşafaka (1863), Dârülaceze (1895), Dârülhayr-1 Ali (1903) et Darüleytam (1914) étaient très importantes. (Bay, 2017 ; Ertem, 1999; Kesgin, 2016; Kongar, 2007; Özbek, 1999; Soydan, 2003; Şafak, 2013; Ünal, 2012; Yıldırım, 1997). Les services fournis dans ces institutions peuvent être considérés comme la base des pratiques modernes de travail social en Turquie. Juste avant la proclamation de la République, la Société Himaye-i Etfal ${ }^{1}$ (1921) a été créée à Ankara pour servir les orphelins et les enfants des soldats tombés au combat. Cette organisation prit plus tard le nom d'Agence Turque de protection de l'enfance (1935) et devint le centre de protection de l'enfance le plus important qui fournissait des services sociaux dans la nouvelle République de Turquie (Duyan et Özgür Bayır, 2016).

Dans cette étude, les pratiques de travail social menées en Turquie après 1923 seront systématiquement discutées dans une perspective chronologique. De nombreuses études ont été publiées sur ce sujet en Turquie. Dans ces études, le développement des services sociaux en Turquie est traité de différentes manières. Par exemple, il est discuté dans une étude comme (1) entre les années 1960 et 1980: c'est le début et le développement du travail social, (2) entre les années 1980 et 2000: c'est l'institutionnalisation et la transformation néo-libérale et (3) après l'an 2000: c'est le conservatisme néolibéral et la compréhension du travail social fondé sur les droits (Şahin Taşğın et Tekin, 2013). Donc,

| 1168 | il y a deux points importants qui distinguent cette étude des études précédentes. Le premier de ces points est que cette étude est traitée dans une structure chronologique différente et discutée avec une perspective critique. Un autre point est que cette étude est rédigée en français, contrairement aux langues

\footnotetext{
${ }^{1}$ Une société de protection des enfants
} 
précédemment publiées (turc, anglais ou allemand). En effet, l'importance de cet article tient au fait qu'il a été écrit pour la première fois afin de créer une littérature française sur l'histoire du travail social après la fondation de la république.

\section{A. ENTRE 1923-1945: PÉRIODE DU PARTI UNIQUE EN TURQUIE}

Lorsque la république a été déclarée en 1923, la nouvelle République de Turquie a également éliminé la compréhension du régime monarchique en cours pendant des centaines d'années en Anatolie. Selon Savran (2016) cette évolution, considérée comme une révolution, est le résultat d'un processus qui a débuté avec les réformes du Tanzimat et de la monarchie constitutionnelle sous l'Empire ottoman. Mais la transition de l'empire d'une structure multinationale à une structure d'une seule nation sera le début d'un processus difficile. La république nouvellement créée doit faire face à de nombreux problèmes économiques, administratifs, politiques, militaires, sociaux et culturels. Ce processus entraînera de nombreuses réformes. Néanmoins, les développements concernant la protection sociale seront discutés dans le cadre de cette étude. Premièrement, nous devons mentionner que la République de Turquie a été fondée par de longues années de guerre et par l'établissement d'une géographie façonnée par la migration forcée à la suite de la dispersion de l'Empire ottoman.

Dans cette période, les pertes humaines et matérielles après la Première Guerre mondiale, le manque d'industrialisation et de croissance économique, des impôts et des revenus étatiques faibles ou irréguliers (Buğra, 2016) ont empêché les efforts nécessaires dans le domaine de la protection sociale et du travail social. Buğra (2016) affirme que la lutte contre la pauvreté n'a pas été considérée comme relevant de la responsabilité de l'État pendant les premières années de l'administration à parti unique (Parti républicain du peuple) en République de Turquie. Selon Buğra (2016), malgré l'approche étatique, les activités de protection sociale étaient largement considérées comme un domaine d'action bénévole et d'organisations de la société civile dans les premières années de la République de Turquie. Pour cette raison, on constate que les organisations bénévoles de l'époque n'agissaient généralement pas indépendamment de l'Etat. Evidemment, malgré le principe de l'étatisme inscrit dans la Constitution depuis 1937, l'Etat n'est pas directement impliqué dans la lutte contre la pauvreté (Buğra, 2016). Au cours de cette période, les premiers efforts du travail social portaient sur la protection de l'enfance. En fait, ces études se poursuivent depuis la période ottomane. Une institution ouverte par Mithat Pacha (1822-1884) pour les orphelins pendant le gouvernement du Danube peut être donnée d'exemple à cet égard.

Un autre exemple est la société Himaye-i Etfal, qui a été fondée à Kırklareli en 1908 et a mené ses activités jusqu'à la guerre des Balkans. Cette association a été rouverte en 1917 sous le nom de la société Himaye-i Etfal à Istanbul (Yildırım, 2012). Avec le début de la guerre d'indépendance turque, les fondateurs de l'association se sont rendus à Ankara. L'association fondée à Ankara du même nom le 30 juin 1921 a reçu le soutien de Mustafa Kemal, qui était alors président de la Grande Assemblée nationale de Turquie (Yolcuoğlu, 2014). La principale différence de cette association par rapport aux autres associations établies à l'époque ottomane est que son champ d'activité et d'intervention ne se limite pas à Ankara. En 1923, la société d'Istanbul Himaye-i Etfal a été fermée en raison du renforcement et de la 
généralisation de la société d'Ankara Himaye-i Etfal2 ${ }^{2}$. En d'autres termes, on peut dire que les deux associations ont été combinées. La société d'Ankara Himaye-i Etfal a commencé à distribuer du lait aux enfants dans le besoin en 1924. En 1927, des salles de bains sanitaires ont été mises en service, que les enfants peuvent utiliser avec leur mère. L'ouverture de jardins d'enfants en 1928, le début des services de soins dentaires en 1929 et l'ouverture d'une école de garde d'enfants à Ankara font partie des activités menées par l'association (Çavuşoğlu, 2001, p. 10). A cet égard, cette association a été l'organisation non gouvernementale la plus active de l'époque en termes d'activités et de services (Ministère de la Famille, du Travail et des Services Sociaux, 2020). En 1935, le nom de l'association a été changé en Agence turque de protection de l'enfance. Le 26 novembre 1937, l'Agence turque de protection de l'enfance a été acceptée en tant qu'association d'utilité publique sur décision du Conseil des ministres (Çavuşoğlu, 2001).

Dans les premières années de la République turque, on constate que différentes activités qui peuvent être considérées aujourd'hui comme des mesures de politique sociale ou des pratiques de travail social ont été menées. Les pauvres vivants dans les villages soutiennent avec la loi sur les villages promulgués en 1924 (Çetin, 1994), une aide et des paiements de pension alimentaire aux familles dans le besoin fournissent avec le code civil promulgué en 1926 (Koçak, 2019). Ces lois sont partie des développements importants de cette période. De même, avec la loi générale sur l'hygiène promulguée en 1930, les pauvres et les orphelins ont bénéficié de services de santé gratuits (Kesgin, 2014). Avec la loi sur la municipalité de 1930, les responsabilités des gouvernements locaux envers les citoyens ont été déterminées. Avec la loi du travail promulguée en 1936, les bases des pratiques de sécurité sociale d'aujourd'hui ont été établies. Le droit de travailler et à la vie des citoyens sont garantis par l'État avec cette loi (Çakmak, 2007). Une aide monétaire régulière a été fournie aux familles des militaires par la loi sur l'aide aux nécessiteux des familles militaires promulguée en 1941. Des aides similaires ont également été apportées à l'époque ottomane; mais avec cette loi, l'aide aux familles des militaires a été transférée aux administrations locales/municipalités (Öztürk, 2013).

Entre 1923 et 1945, les études sur les services de protection sociale ainsi que toutes les politiques et pratiques générales ont été déterminées par le Parti républicain du peuple. Au cours de cette période, divers rapports, publications, conférences ou projets ont été réalisés en plus des réglementations juridiques et des efforts d'institutionnalisation mentionnés jusqu'à présent concernant les services de protection sociale. Le "projet des enfants vagabonds" lancé en 1942 peut être cité comme exemple de ces développements. La Turquie, en tant que pays ne participant pas à la Seconde Guerre mondiale, provoquée par la guerre, a été contrainte de faire face à de nombreux problèmes. En particulier, les problèmes économiques et la pauvreté causés par ces problèmes ont marqué les années 1940. Pendant

| 1170 | 2 Pendant cette période, des développements très importants ont eu lieu; mais en raison de l'objectif de l'étude, des informations détaillées ne peuvent être incluses dans cette section. Par exemple, le 21 mars 1923, le Dr. Fuat Umay, le président de la société Himaye-i Etfal et le député de Bolu, se rendit aux États-Unis pendant cinq mois pour recueillir des dons auprès des Turcs qui y vivaient, avec l'autorisation de la Grande Assemblée nationale de Turquie. À la suite de ce développement avec le congrès tenu à l'hôtel Alpin le 15 avril 1923, la branche centrale de la société Himaye-i Etfal a été ouverte à New York.

Pour des informations détaillées, visitez https://ailevecalisma.gov.tr/chgm/hakkimizda/tarihce/ [Date d'accès: 12.05.2020] 
cette période, le nombre croissant de pauvres ou d'orphelins dans les rues d'Istanbul figurait parmi les questions prioritaires du gouvernement.

En 1942, le président İsmet İnönü pensait qu'il était nécessaire d'intervenir directement dans cette situation. Ainsi, il a eu une réunion avec Dr. Fethi Erden, membre de l'Agence de protection de l'enfance. A l'issue de cette réunion, toutes les installations de l'Etat, notamment la présidence, ont été affectées à la résolution de ce problème (Burgaç, 2018). Bref, un état de mobilisation a été déclaré. Un questionnaire a été préparé dans le cadre du "projet des enfants vagabonds" et appliqué aux enfants errant ou travaillant librement dans les rues d'Istanbul. Dans le questionnaire, des questions étaient posées sur l'état de l'enfant, la situation de la mère et du père et l'état général de la famille (Sarıkaya et Çavuşoğlu, 2014). La méthode et l'approche suivies dans ce projet nous rappellent la personne dans un environnement dominant dans les pratiques de travail social d'aujourd'hui. Les enfants ont été étudiés dans des contextes micro et mezzo et des suggestions de solutions ont été essayées pour être développées.

En 1944, diverses études ont commencé à la Grande Assemblée nationale de Turquie pour soutenir les enfants négligés et errants. À la suite de ces études, un rapport a été soumis à la présidence de l'Assemblée en 1945. Lors de l'examen de ce rapport, les enfants de Turquie se sont divisés en différentes catégories telles que les pauvres, les sans-abri, les criminels, les illégitimes ou les apprentis (Çavuşoğlu et Çetin, 2019). De telles catégories peuvent paraître inacceptables aujourd'hui, lorsque les enfants défavorisés sont traités comme ayant besoin de protection. Cependant, une telle catégorisation peut être comprise à un moment où la Convention internationale des droits de l'enfant (CIDE), voire la Déclaration universelle des droits de l'homme (DUDH) des Nations Unies, n'a pas encore été publiée. En fait, ces rapports et analyses constitueront la base de la première loi sur les enfants ayant besoin de protection en Turquie, qui a été adoptée par la Grande Assemblée nationale turque en 1949.

\section{B. ENTRE 1946-1979: PÉRIODE INITIALE DE PRATIQUE PROFESSIONNELLE DU TRAVAIL SOCIAL EN TURQUIE}

Cette période doit être considérée comme une période qui façonnera le 21e siècle pour le monde entier. En effet, un nouveau parti a été fondé en 1946 sous le nom de Parti démocrate en Turquie. Dans le monde, la Seconde Guerre mondiale a pris fin et une nouvelle ère a commencé à s'appeler la guerre froide. Pendant cette période; les Nations Unies (ONU) ont été créées, la Déclaration universelle des droits de l'homme des Nations Unies a été acceptée, le Pacte de Varsovie a été signé et la guerre spatiale a commencé. Évidemment, ces développements sont importants et viennent à l'esprit en premier. En dehors de ceux-ci, de nombreux développements mondiaux, continentaux, nationaux ou régionaux qui changeront la vie des citoyens en Turquie ont également été vécus. Cependant, selon la nature de cette étude, seuls les événements importants pour le développement du travail social en Turquie seront discutés.

Au cours de cette période, la première loi sur la protection de l'enfance de la République de Turquie, la loi $n^{\circ} 5387$ sur les enfants ayant besoin de protection a été adoptée (27 mai 1949). Ainsi, l'État a pris pour la première fois la responsabilité directe de la protection des enfants. Selon la loi, les enfants ayant besoin de protection doivent avant tout être bien élevés. Pour cela, ils doivent d'abord être éduqués 
pour exercer une profession. Différentes institutions et individus tels que les chefs de village, les conseils des anciens/sages du village, les municipalités, le ministère de la Santé et de l'Aide Sociale et le ministère de l'Éducation Nationale ont été désignés à cet égard. Les enfants qui n'ont pas de logement et qui n'ont pas d'âge scolaire seront placés dans des dortoirs qui seront ouverts par le ministère de la Santé et l'Aide Sociale. Les enfants d'âge scolaire resteront dans les dortoirs qui seront ouverts par le ministère de l'Éducation Nationale.

Dans le cadre de cette loi, des règlements ont été établis pour la première fois sur des questions telles que l'adoption d'enfants séjournant à l'Agence turque de protection de l'enfance ou leur placement dans une famille d'accueil. De plus, il a été décidé de créer des commissions de protection de l'enfance dans les centres provinciaux ou quartiers (Çavuşoğlu et Çetin, 2019). En 1957, certains articles de la loi sur la protection de l'enfance précédemment promulguée ont été modifiés et une nouvelle loi sur les enfants ayant besoin de protection $n^{\circ} 6972$ a été adoptée. Ainsi, les services pour les enfants ayant besoin de protection ont été transférés à ces commissions de protection nouvellement créés et donc localisés (Çağlar, 1982). Cependant, il y a un point qui ne doit pas être ignoré ici. Ces lois ne sont pas suffisantes pour prévenir la pauvreté des enfants ou améliorer le bien-être des enfants en Turquie. Lorsqu'on examine des études académiques de cette période relative à la pauvreté des enfants, on voit généralement que ces enfants discutent dans le contexte de l'inconfort causé à l'environnement et aux autres (Çağlar, 1982; Buğra, 2016).

Ces années, qui était un parti démocrate nouvellement établi au pouvoir en Turquie, et était une période où différents partis politiques doivent être établis. Cette période peut être acceptée en même temps que les premières années de pratique professionnelle du travail social en Turquie. Un projet de loi sur la création d'un institut des services sociaux en Turquie a été élaboré en 1955. En effet, les études sur cette loi ont commencé en 1954. Selon Buğra (2016), la création d'un institut de services sociaux en Turquie a commencé avec des activités des Nations Unies. En 1959, il a été décidé de créer une "Académie des services sociaux" qui appartient au ministère de la Santé et de l'Aide Sociale en Turquie. Pour cela, en vertu de la loi $n^{\circ} 7355$, l'article 1, portant création de l'Institut des services sociaux, a été publié par la Grande Assemblée nationale de Turquie. Sur la base de cette loi, l'Académie des services sociaux a été fondée à Ankara en 1961 (Güzel, 2017). Il n'y a eu aucun autre développement qui puisse être évalué en termes de pratiques de travail social pendant la période du Parti démocrate. Il est critiqué que le Parti démocrate n'ait pas été à la hauteur dans la lutte contre la pauvreté à cette période où les politiques keynésiennes ont été mises en œuvre comme dans les pays occidentaux (Buğra, 2016). Cependant, il ne faut pas ignorer que les pays occidentaux sont des pays développés et ils ont commencé à s'industrialiser au XIXe siècle. Donc, ils avaient besoin de main-d'œuvre pour se développer à nouveau après la Seconde Guerre mondiale.

| 1172 | En effet, la pauvreté en Turquie n'a jamais été considérée comme une condition liée au développement et à la commercialisation capitaliste jusqu'à cette époque. La déclaration du Premier ministre Adnan Menderes de l'époque, "Dans notre pays, la justice sociale est une affaire d'agriculteur et de village plutôt qu'une question de travail comme dans d'autres pays" (Buğra, 2016), est une preuve que le Parti démocrate traite la pauvreté dans l'axe de ceux qui vivent dans le village. Ironiquement, à cette 
époque, la politique qui a amené le Parti démocrate au pouvoir était aussi la politique économique libérale, qui était anti-étatiste. À cet égard, des principes importants et positifs ont été inclus dans le programme gouvernemental du Parti démocrate, notamment pour protéger les intérêts des travailleurs. Même dans les règlements adoptés, on voit que le Parti démocrate a une approche positive envers les travailleurs dans le cadre de la politique de l'État social (Kanca, 2012).

Après le coup d'État militaire du 27 mai en 1960, la Constitution adoptée en 1961 est un tournant important dans la pratique du travail social en Turquie. Lorsque cette constitution est examinée, l'accent est mis sur le fait que l'État doit assumer ses responsabilités en matière sociale (Gözler, 1999). Les droits et problèmes sociaux sont mis en évidence pour la première fois dans cette constitution. Dans le deuxième article de la constitution, la République de Turquie est définie comme un "État de droit social". La définition en question confirme cette approche sociale. Pour cette raison, on peut dire que la Constitution de 1961 présente des caractéristiques similaires aux politiques keynésiennes mises en œuvre en Europe occidentale. Selon Kongar (2007), la Constitution de 1961 a une perspective extrêmement avancée du travail social. Une grande importance est attachée aux services de santé, de logement et d'éducation. En plus de ce service, la famille et les enfants ayant besoin de protection ont été pris en charge, ce qui est très important pour l'avenir des services sociaux en Turquie.

Les années 1960 ont été une année très troublée et difficile pour la Turquie. De nouveaux partis ont commencé à se créer dans l'environnement politique qui a commencé à se relancer après le coup d'État militaire. En plus de la transformation politique, un changement sociologique a commencé à se produire. Pendant cette période, des mouvements migratoires à l'intérieur et à l'extérieur du pays ont commencé à se produire. Les pauvres et les paysans du pays ont émigré vers les villes et ont commencé à créer de nouveaux espaces urbains. Ainsi, l'environnement politique changeant et la structure démocratique diversifiée ont gagné de nouveaux électeurs (Kesgin, 2014). Des millions de citoyens turcs ont immigré dans les pays d'Europe occidentale par des accords de travail signés avec des pays tels que l'Allemagne, la France, les Pays-Bas, la Belgique et l'Autriche. Ainsi, les envois de fonds des migrants ont joué un rôle important dans le développement de la Turquie.

Au cours de cette période, il y a deux développements plus importants en termes de pratique du travail social en Turquie. Le premier d'entre eux est la création de la Direction générale des services sociaux au sein du ministère de la Santé et de l'Aide Sociale en 1963. Donc, tous les services d'assistance sociale et de sécurité sociale en Turquie, tels que les enfants vulnérables, la fourniture de services de soins et de réadaptation pour les handicapés et les personnes âgées, ont été transférés à cette direction nouvellement créée. En outre, la responsabilité de répondre aux besoins de sécurité sociale des pauvres et des démarches administratives et scientifiques de l'Académie des services sociaux a été transférée à cette direction (Çavuşoğlu et Çetin, 2019). Ces années sont également les années de transition vers les plans quinquennaux de développement en Turquie.

Le premier plan quinquennal de développement a commencé à être mis en œuvre entre 1963 et 1967. Dans ce premier plan, le but du travail social est exprimé comme l'élimination des problèmes sociaux et des irrégularités qui se produisent dans divers domaines. En conséquence, on peut affirmer que le travail social est géré dans un domaine très limité et incertain dans ce plan. Cependant, lorsque le 
plan est examiné en détail, on constate que les problèmes de cette définition sont classés comme (1) les enfants ayant besoin de protection, (2) les maisons de retraite pour personnes âgées et (3) les centres de réadaptation (Kongar, 2007). L'approche la plus innovante pour les pratiques de travail social dans le plan est d'assurer la coordination entre les organisations bénévoles (non gouvernementales) et les institutions publiques et de créer des "conseils de coordination" au niveau local pour un travail conjoint. Pendant cette période, les volontaires des organisations turques (société civile) étaient bien moins nombreux qu'aujourd'hui et étaient rassemblés dans certaines provinces. Cette situation a empêché les activités ou études de se généraliser au niveau national. De plus, les conseils de coordination créés au cours de ce processus n'ont pas été efficaces car ils n'étaient pas officiellement supervisés et soutenus (Kongar, 2007).

On voit que la deuxième (1968-1972) et la troisième (1973-1977) plan quinquennal de développement ont également mis l'accent sur les pratiques de travail social. Lorsque l'on compare le deuxième plan quinquennal de développement au premier plan, on comprend qu'il s'agit d'un plan plus significatif et scientifique. Dans le deuxième plan quinquennal de développement, la déclaration "les besoins de sécurité sociale qui surgissent avec l'industrialisation et la modernisation et qui ne peuvent être satisfaits par les assurances sociales seront satisfaits par les services de protection sociale" (Organisation de Planification d'État, 2020) souligne le principe adopté pour les services sociaux. Dans le deuxième plan, les services de protection sociale ont été considérés comme relevant directement de la responsabilité de l'État et des objectifs plus réalistes et réalisables ont été déterminés conformément au premier plan. Dans ce plan, il est très important que la responsabilité des pratiques de travail social soit transférée au gouvernement central plutôt qu'aux administrations locales et aux organisations bénévoles (non gouvernementales). Les pratiques de travail social dans ce plan comprennent des activités et des programmes tels que l'assistance sociale pour les personnes âgées et les pauvres, la protection de l'enfance et de la jeunesse, les soins à la famille, le soutien psychologique, les soins aux handicapés, la réadaptation médicale et sociale, les services aux criminels et les activités de loisirs. La perspective la plus innovante de ce plan est l'idée que les pratiques d'assurance sociale et de travail social devraient être intégrées dans un système de sécurité sociale (Organisation de Planification d’État, 2020).

En fait, un autre développement important dans le domaine de la protection sociale au cours de cette période a été réalisé avec la promulgation de la loi sur l'assurance sociale en 1964. Avec cette loi, le nom d'Institution d'assurance des travailleurs a été changé en Institution de sécurité sociale. Donc, la sécurité sociale a été retirée du statut de droit spécifique seulement aux travailleurs et a été tentée de se transformer en une structure applicable au grand public. En plus de cette institution, l'Agence d'assurance sociale des travailleurs indépendants a été créée en 1972, connue sous le nom de "Bag-Kur". Les citoyens turcs travaillant indépendamment comme les commerçants, les agriculteurs et les femmes au foyer qui ne sont soumis à aucune institution de sécurité sociale ont le droit d'être assurés auprès de cette institution (Institution de sécurité sociale, 2020). Selon L'organisation Internationale du Travail (2003), "la sécurité sociale est la protection qu'une société offre aux personnes et aux ménages pour garantir l'accès aux soins de santé et la sécurité du revenu, surtout en cas de vieillesse, de maladie, d'invalidité, d'accident du travail, de maternité ou de disparition du soutien de famille". Comme on peut le voir clairement dans cette définition, la sécurité sociale ne couvre pas uniquement le domaine lié à la 
santé. Toutes sortes de problèmes et de négativités qui aggraveront les conditions de vie des hommes ou réduiront leur niveau de vie sont abordées dans le cadre du concept de sécurité sociale. Ainsi, les réformes de la sécurité sociale menées en Turquie dans les années 1960-1970 doivent être évaluées dans cette perspective.

En 1976, une loi a été adoptée pour les citoyens de plus de 65 ans, qui n'étaient pas en mesure de prendre soin d'eux-mêmes, qui n'avaient pas de personne pour s'occuper d'eux, de revenu mensuel, d'assurance maladie, de pension alimentaire et de revenu (Kesgin, 2016). Cette loi est très populaire en ces jours et connue sous le nom de loi $\mathrm{n}^{\circ} 2022$ dans la société. Aujourd'hui, non seulement les salaires des nécessiteux, des faibles et des orphelins de plus de 65 ans, mais aussi des frais de prise en charge des personnes handicapées sont payés dans le cadre de cette loi. Cependant, il ne faut pas oublier que de graves problèmes ont été rencontrés dans la mise en œuvre des réglementations juridiques susmentionnées au cours de ces années. Les développements à l'échelle mondiale au cours de cette période ont profondément affecté la Turquie. Par exemple, la crise pétrolière (crise de stagflation) en 1973 a touché de nombreux pays. Du fait de cette crise, la structure sociale de l'État a été remise en cause dans les pays occidentaux, qui ont adopté des politiques keynésiennes depuis 1945. Dans la période suivante, les pratiques de l'État social dans les pays occidentaux ont perdu de leur importance et cette structure administrative est entrée dans une période de déclin. En fin de compte, l'instabilité de l'économie, qui a été façonnée par l'intervention de l'État entre 1945 et 1970, et les évolutions négatives des taux d'inflation et des investissements ont créé une opportunité pour des politiques néolibérales, qui ont défendu que l'État devrait réduire les dépenses publiques (Gümüş, 2010).

\section{ENTRE 1980-2000: TRANSFORMATION NÉOLIBÉRALE ET PRATIQUES D'INSTITUTIONNALISATION}

Les politiques néolibérales qui ont commencé à être mises en œuvre après 1980 sont fondées sur l'idée que la distribution des revenus peut être réalisée par le meilleur mécanisme du marché. Cette idée implique une compréhension qui repousse l'intervention de l'État dans la répartition des revenus. Limiter l'intervention de l'État signifie la perturbation des pratiques de protection sociale offertes directement par l'État. À la fin des années 1970, la part des dépenses sociales des pays de l'OCDE dans les dépenses publiques dépassait 50\%, à l'exception de l'Angleterre (Artan, 2012). Avec l'adoption de politiques néolibérales, en particulier les pays occidentaux avancés et les pays en développement comme la Turquie, ont également commencé à perdre de nombreuses acquisitions importantes pour les politiques de protection sociale. Dans cette nouvelle période après 1980, le rôle de l'État concernant de services de protection sociale est resté à l'arrière-plan, tandis que la participation d'organisations bénévoles (non gouvernementales) et du secteur privé est apparue au premier plan. Au cours de cette période, on constate que de nouvelles définitions telles que "état favorable au marché" ont été élaborées pour définir le rôle de l'État. Il vise à fournir autant que possible des services publics par le secteur privé et des organisations bénévoles (non gouvernementales) sous le contrôle de l'État. Tous ces changements ont révélé différentes applications telles que la privatisation, la décentralisation et le pluralisme de la protection sociale dans la fourniture de services de protection sociale dans l'administration publique (Artan, 2012). 
Les années 1980 ont été une période difficile pour la Turquie. Tout d'abord, en raison des conflits politiques et des événements sociaux qui durent depuis les années 1970, le coup d'État militaire du 12 septembre 1980 a eu lieu. La nouvelle constitution préparée par l'administration militaire a été adoptée en 1982. Cette constitution est très importante pour déterminer les progrès accomplis dans l'exécution des services de protection sociale en Turquie les années à venir. À l'instar de la Constitution de 1961, la Constitution turque de 1982 met l'accent sur «un état de droit social» et donne aux citoyens certains droits sociaux et économiques. Néanmoins, la pensée que ces droits sociaux coûteraient à l'État et à l'économie limitait le principe de l'État social et le laissait derrière les priorités financières et économiques (Karaca et Çam, 2019). On voit que la compréhension de l'État social a été un peu élargie dans la Constitution turque de 1982. En outre, il existe de nouvelles dispositions sur la sécurité sociale, l'assistance sociale et les droits sociaux. Particulièrement à l'article 17, il est dit que "chacun a le droit de vivre, de protéger et de développer son existence matérielle et spirituelle" et ici la responsabilité est attribuée à l'État (Constitution turque de 1982; article 17, 2020).

Dans la Constitution turque de 1982, la sécurité sociale et l'assistance sociale sont traitées dans un cadre large. Sur la base de cette constitution, de nombreuses lois sur l'assistance sociale ont été adoptées. Cependant, lorsqu'on considère les droits sociaux, on constate que ces droits sont traités plus clairement dans la Constitution de 1961 que dans la Constitution turque de 1982. Les lois adoptées sur la base de la Constitution turque de 1982 sont très importantes pour l'institutionnalisation du travail social en Turquie. En effet, les nouvelles institutions créées au cours de cette période et elles ont considérablement accéléré le développement du travail social en Turquie. À ce stade, en particulier la loi sur les services sociaux et l'institution de protection de l'enfance $\left(n^{\circ} 2828\right)$ et la loi sur la promotion de l'assistance sociale et de la solidarité $\left(n^{\circ} 3294\right)$ sont très importantes.

La loi sur les services sociaux et l'institution de protection de l'enfance a été adoptée en 1983 sur la base des articles de la section intitulée "Droits et devoirs sociaux et économiques" de la troisième partie de la Constitution turque de 1982. Dans la troisième partie de la Constitution turque de 1982, de nombreux droits tels que la protection de la famille, les droits des enfants, le droit à l'éducation, le droit au travail, le droit à la négociation collective, le droit à une répartition équitable des salaires, le droit à la santé, le droit à l'environnement et au logement, le droit à la sécurité sociale; la protection des jeunes, des personnes handicapées, des anciens combattants et des personnes âgées ont été reconnus. L'État a été défini comme responsable de garantir ces droits. Avec ces lois et règlements, le travail social a atteint une structure nouvelle et différente en Turquie. Cet événement est le deuxième développement significatif dans le domaine du travail social après la loi adoptée sur la création de l'Académie des services sociaux en Turquie (Artan, 2012). Avec cette loi, les pratiques de travail social ont acquis une dimension holistique et juridique. En outre, la Direction générale des services sociaux et l'Agence turque de protection de l'enfance ont été fusionnées, et puis dénommées Agence des services sociaux et de la protection de l'enfance (SHÇEK). L'importance des notions de famille et d'enfants ayant besoin de protection dans cette loi a ouvert la voie à des activités de protection, de prévention et de soutien pour la famille et les enfants. Ainsi, les services offerts par différentes institutions et organisations pour les enfants ayant besoin de protection ont été essayés d'être rassemblés sous un même toit. L'agence des services sociaux et de la protection de l'enfance est affiliée au Premier ministère et fonctionne en tant 
qu'institution relevant directement du Premier ministre. Cette institution a fonctionné jusqu'à la création du ministère de la Famille et des Politiques Sociales en 2011.

Quand nous sommes arrivés en 1986, la loi sur la promotion de l'assistance sociale et de la solidarité ( $n^{\circ}$ 3294) a été adoptée. Dans le cadre de cette loi, des fondations d'assistance sociale et de solidarité ont été créées dans chaque province et district. Le contrôle de ces fondations était confié aux gouverneurs de province et aux gouverneurs de district (qaimaqam). Un "conseil d'administration de la fondation" a été établi pour les processus de gestion et de prise de décision de chaque fondation. À cet égard, nous pouvons considérer les fondations en question comme le reflet de l'évolution de l'approche de l'administration publique. En fait, les autorités centrales de l'administration publique ont été transférées aux gouvernements locaux et ont adopté une structure décentralisée. Désormais, conformément au principe de subsidiarité, il vise à fournir des services de protection sociale aux clients de l'unité de gestion la plus proche. D'autre part, ce nouveau système d'assistance sociale, qui repose fortement sur les dons et le volontariat comme ressources économiques et humaines, peut également être considéré comme le résultat naturel de la néo-libéralisation. Depuis la création, les fondations d'assistance sociale et de solidarité ont fourni des aides en espèces et en nature dans différents domaines tels que la nourriture, le chauffage, l'hébergement, la santé, l'éducation et les soins. Toutes les fondations d'assistance sociale et de solidarité sont rattachées directement au Premier ministère au plus haut niveau. En fait, cette pratique a été critiquée de temps en temps au motif qu'elle était utilisée comme outil de propagande du parti du Premier ministre en période électorale. Cette pratique s'est poursuivie jusqu'à la création de la Direction générale de l'assistance sociale et de la solidarité en 2004. Plus tard, avec la création du ministère de la Famille et des Politiques Sociales en 2011, elle est devenue une direction générale au sein du ministère.

L'Agence des services sociaux et de la protection de l'enfance et les fondations d'assistance sociale et de solidarité sont les deux principales institutions et organisations qui fournissent du travail social dans toutes les provinces et districts jusqu'à la création du ministère de la Famille et de la Politique Sociale en Turquie. Pendant cette période, de nouvelles institutions ont également été créées pour opérer dans le domaine du travail social, même si elles n'avaient pas de bureaux dans chaque province et district. L'institution de recherche familiale créée en 1989 et l'Administration pour les personnes handicapées créée en 1997 peuvent être citées comme exemples de ces institutions. Ils sont créés pour mener des activités et fournir du travail social aux familles et aux personnes handicapées en Turquie. Entre 1980 et 2000, les développements en Turquie dans le domaine du travail social ne se limitent pas à ces activités d'institutionnalisation. Toutes les organisations non gouvernementales après le coup d'État militaire de 1980 en Turquie ont été fermées. L'association d'anciens élèves de l'académie des services sociaux, créée en 1972, est l'une des associations fermées. En 1987, cette association a été rétablie sous le nom d'Association des travailleurs sociaux (SHUDER). L'association a commencé ses activités en 1988 et les membres fondateurs de l'association sont des membres du corps professoral de l'École des services sociaux de l'Université Hacettepe (Bozkurt, 2019). Cela montre à quel point l'académie était si efficace et si importante dans le processus d'organisation du travail social professionnel en Turquie. Parmi les objectifs fondateurs de cette association, celui de "promouvoir la profession de travailleur social et contribuer à son développement" (Association des travailleurs sociaux, 2020) est le premier. Cet objectif révèle l'état actuel des services sociaux en Turquie dans les années 1980. Pendant cette période, il y a un 
besoin de reconnaissance et d'acceptation dans la sphère publique et sociale pour le travail social en Turquie.

Enfin, bien que cela n'ait pas d'impact direct sur la pratique du travail social en Turquie pendant cette période, il vaut mieux parler d'un événement qui affectera indirectement après les années 2000. Comme on le sait, après le sommet de Luxembourg de 1997, la Turquie a été suivie d'une période politique de suspension de toutes les relations avec l'Union européenne (UE). Cependant, lors du sommet d'Helsinki en 1999, la candidature de la Turquie à l'UE a été acceptée par les décisions prises et a annoncé que le document de partenariat d'adhésion serait préparé. Par la suite, les relations bilatérales ont été relancées et une nouvelle ère a été introduite, où les obligations et intérêts mutuels ont été discutés (Ateş, 2019). Les effets de cette période sur le travail social sont apparus après les années 2000.

\section{LES ANNÉES 2000 ET AU-DELÀ: LE PROCESSUS DE CANDIDATURE DE LA TURQUIE À L'UE, LES EFFORTS DE REGROUPEMENT DE TOUTES LES PRATIQUES DE TRAVAIL SOCIAL SOUS UN SEUL MINISTÈRE ET LA FOLIE DE LA FORMATION DES TRAVAILLEURS SOCIAUX}

Lors du sommet d'Amsterdam organisé par l'UE aux Pays-Bas en 1997, le document «Agenda 2000» sur les problèmes à discuter et l'élargissement de l'agenda de l'UE au XXIe siècle a été adopté. Dans ce document, le nom de la Turquie était impliqué de quelque manière que ce soit dans la section sur l'élargissement de l'UE (Alkan, 2013). Cependant, la Turquie était un pays prêt à négocier un candidat à l'UE en 2000. D'un autre côté, la Turquie est engagée dans une instabilité politique et une crise économique continues depuis de nombreuses années. De plus, en août 1999, le tremblement de terre de Marmara s'est produit et le pays est confronté à de graves problèmes économiques, politiques et sociaux. Ces développements ont été les précurseurs de l'an 2000 sera difficile pour la Turquie. Mais le fait que la Turquie, pays candidat à l'UE, était aussi un moyen de sortir de la crise.

La Turquie, en particulier immédiatement après le sommet d'Helsinki, a commencé à prendre des mesures importantes dans l'arène politique et est entrée dans la période de réformes intensives. Ces développements ont préparé le terrain pour le développement d'une relation positive entre l'UE et la Turquie. La Turquie a accepté de supprimer les huit paquets de réformes différents contenant un grand nombre de lois et de règlements afin de se conformer aux critères politiques de l'UE. Parmi ces paquets d'harmonisation, il y avait aussi des règlements qui nécessitaient des changements dans la Constitution turque de 1982 (Gözen, 2006). Dans ce processus, il y a eu de nombreux développements importants en Turquie pour le développement du travail social; mais le développement le plus important a été la promulgation du Code civil, qui a été amendé en 2001 (après 75 ans plus tard). Des changements importants ont été apportés au nouveau Code civil pour l'égalité entre les femmes et les hommes et la protection de la famille, des enfants, des handicapés et des orphelins. En outre, d'importantes réglementations ont été introduites dans cette loi concernant la création et le fonctionnement des associations et des fondations (Code civil turc, 2001). Dans le troisième paquet d'harmonisation en 2002, de nouvelles réglementations ont été adoptées concernant la loi sur les associations et les fondations en plus du Code civil (Altıntaş, 2008). Ces réglementations formeront également la base de la pratique du travail social dans les années à venir en Turquie. 
L'évolution du processus de réforme intensif ne se limite pas aux paquets d'harmonisation. Le Parti de la justice et du développement (AKP), qui est arrivé au pouvoir en tant que parti unique à la suite des élections générales tenues en 2002, a pris des dispositions juridiques importantes dans le domaine du travail social au cours des années suivantes. L'année 2005 est particulièrement importante dans ce processus. La loi sur la protection de l'enfance ( $\left.n^{\circ} 5395\right)$, la loi sur les personnes handicapées $\left(n^{\circ} 5378\right)$ et la loi sur la municipalité de 2005 ( $n^{\circ}$ 5393), qui comprennent des mesures et des pratiques de protection des enfants, des femmes, des personnes âgées et des handicapés, ont été adoptées.

Avec la loi sur la protection de l'enfance ( $\mathrm{n}^{\circ}$ 5395), la protection des enfants ayant besoin de protection ou poussés au crime, la garantie des droits des enfants et la fourniture de services de soutien ont été transférées à la responsabilité de l'Agence des services sociaux et de la protection de l'enfance. En outre, il était rassemblé et réglementé par cette loi que des mesures de conseil, de soins, d'hébergement, de santé et d'éducation devraient être prises pour protéger ces enfants dans l'environnement familial. Il est prévu que le ministère de la Justice, le ministère de l'Éducation Nationale, le ministère de la Santé et l'Agence des services sociaux et de protection de l'enfance (SHÇEK) agissent en coordination par cette loi. De plus, il a été garanti pour la première fois qu'au moins un travailleur social est affecté aux tribunaux de l'enfance et de la famille affiliés au ministère de la Justice et qu'un rapport d'enquête social (SİR) est préparé pour les enfants (Artan, 2012).

Avec la loi sur les personnes handicapées ( $n^{\circ} 5378$ ), il existe des règles pour la prise en charge des personnes handicapées qui ont besoin de soins dans leur famille ou dans des institutions de soins. Cette loi prévoit un soutien économique pour les soins à domicile ou les soins payants dans les établissements privés. L'Agence des services sociaux et de la protection de l'enfance est également chargée de recevoir, d'évaluer et de finaliser les demandes de ces personnes (Artan, 2012). Les personnes handicapées en Turquie ont commencé à être classées en fonction de leur handicap. Ainsi, il leur est devenu possible de bénéficier des services qui leur conviennent. En outre, cette loi comprend des règlements sur la manière dont les services tels que les soins, la réadaptation, l'emploi et la formation seront fournis aux handicapés. La loi sur les personnes handicapées ( $n^{\circ} 5378$ ) vise principalement à garantir l'égalité des chances en matière de handicap, à protéger les droits de l'homme et à prévenir la discrimination. Cette approche montre que l'adoption d'une perspective post-moderne en termes de services pour les personnes handicapées en Turquie. Cependant, lorsque les pratiques menées à ce jour et la perspective et la participation de la société sont prises en considération, on constate qu'un bon développement n'a pas encore été réalisé.

Bien que la loi sur la municipalité de 2005 ( $n^{\circ}$ 5393) ne soit pas une loi directement liée au travail social, certains articles de la loi concernent les devoirs et les responsabilités des municipalités en matière de services sociaux. Par exemple, à l'article 14 de la troisième section, qui porte sur les devoirs et responsabilités des municipalités, il est mentionné que les municipalités métropolitaines et les municipalités de plus de 100000 habitants doivent ouvrir des foyers pour les femmes et les enfants. Il est mentionné que les municipalités de moins de 100000 habitants peuvent ouvrir des foyers pour les femmes et les enfants en évaluant leur situation financière et les priorités de service (Loi sur la municipalité de 2005,2020). Dans l'article 60 de la cinquième section relative aux revenus et dépenses des 
municipalités, "les services sociaux et les aides à fournir aux pauvres, aux nécessiteux et aux orphelins et aux handicapés" sont inscrits parmi les dépenses de la municipalité. Dans le 77e article relatif à la participation volontaire aux services municipaux, la municipalité est chargée d'assurer la solidarité et la participation aux services tels que la santé, l'éducation, les sports, l'environnement, les services sociaux et l'assistance. De même, on observe que les municipalités sont responsables de la mise en œuvre des programmes de participation de volontaires afin d'accroître l'efficacité et la productivité dans la fourniture de services aux personnes âgées, aux femmes, aux enfants, aux handicapés, aux pauvres et aux orphelins (Loi sur la municipalité de 2005, 2020).

Les années 2000 sont assez importantes en termes d'applications de travail social en Turquie, non seulement en termes d'introduction d'une nouvelle législation, mais aussi en termes de développement de l'enseignement du travail social et de la formation des travailleurs sociaux. Jusqu'au début des années 2000, il n'existait qu'un département de travail social à l'Université Hacettepe en Turquie. En 2002, le pays candidat de la Turquie à l'UE avait créé un environnement idéal pour l'ouverture de nouveaux départements de travail social. Mais en même temps, l'impact de l'évolution de la conjoncture politique de la Turquie (Parti de la justice et du développement est arrivé au pouvoir) ne doit pas être ignoré (Alptekin, Topuz et Zengin, 2017). Ainsi, pour la première fois, un département de travail social a été ouvert à l'Université Başkent d'Ankara en 2002. Au cours de la période suivante, des départements de travail social ont été ouverts à l'Université Selçuk (Konya), à l'Université d'Adnan Menderes (Aydın), à l'Université Sakarya et à l'Université Süleyman Demirel (Isparta). Ensuite, les départements de travail social ont été ouverts à l'Université d'Ankara en 2007, à l'Université Yalova, à l'Université Düzce et à l'Université Gümüşhane en 2009, à l'Université Kocaeli et à l'Université Maltepe en 2010 (Güzel, 2017). Aujourd'hui, plus de 60 universités dispensent une formation en travail social en Turquie.

Cette croissance rapide et incontrôlée sur une courte période a suscité diverses critiques. Ces critiques s'adressent principalement au département de travail social de l'Université Hacettepe, qui mène des activités éducatives en tant que département unique depuis de nombreuses années. Ce département est critiqué pour ne pas avoir développé de stratégies pour gérer le processus qui a eu lieu après les années 2000 et pour ne pas avoir dirigé le personnel académique du département vers différentes universités (Alptekin, 2016). Cependant, ce département n'est évidemment pas le seul facteur dans ce processus. Le Conseil de l'enseignement supérieur turc (YÖK) est également responsable de la planification, du fonctionnement et de la coordination des universités et du système d'enseignement supérieur en Turquie. Le Conseil de l'enseignement supérieur turc fait l'objet de sérieuses critiques en ce qui concerne l'enseignement du travail social; car il n'a pas été en mesure de faire la planification nécessaire et permet que l'enseignement du travail social soit dispensé dans des facultés d'éducation ouvertes.

| 1180 | Aujourd'hui, trois universités offrent une formation en travail social à distance ou en ligne. Des milliers d'étudiants sont diplômés de ces universités chaque année en suivant une formation en travail social à distance ou en ligne (Conseil de l'enseignement supérieur turc, 2020). Dans ce cas, malheureusement, cela conduit à remettre en question la compétence des travailleurs sociaux en Turquie. En conséquence, ces années se sont écoulées dans la précipitation et l'effort de diplômés des travailleurs 
sociaux en Turquie en termes de formation en travail social. Par conséquent, les personnes, institutions et organisations liées à ce processus sont exposées à diverses critiques.

Si nous mettons de côté la formation en travail social, on constate que les pratiques du travail social ont été menées par différentes institutions et organisations en Turquie dans les années 2010. L'agence des services sociaux et de la protection de l'enfance en tant qu'organisme responsable de la pratique du travail social ou des services sociaux en Turquie depuis 1983. Malheureusement il n'y a aucun intérêt sauf pour les services aux enfants. Cette situation a été critiquée pour ne pas rassembler ces services sociaux sous un même toit. Suite à ces critiques, le ministère de la Famille et des Politiques Sociales a été créé en 2011 et toutes les activités de travail social ont été regroupées sous ce ministère (Kesgin, 2016). Grâce à ce ministère les enfants, les femmes, les personnes handicapées et âgées, les services familiaux et sociaux et les demandes de travail social ont été rassemblés dans une seule institution.

Au sein de ce ministère, il existe cinq services différents: (1) Direction générale des services familiaux et communautaires, (2) Direction générale des services à l'enfance, (3) Direction générale des services aux personnes handicapées et âgées, (4) Direction générale du statut des femmes et (5) Direction générale des aides sociales. En fait, on sait que ces services fonctionnaient sous différents ministères ou le premier ministère au cours des périodes précédentes. Par exemple, la Direction générale des services familiaux et communautaires est le prolongement de l'Institution de recherche familiale créée en 1989. De même, la Direction générale des services aux personnes handicapées et âgées est le prolongement de l'Administration des personnes handicapées créée en 1997. Depuis 2011, des organisations provinciales du ministère de la Famille et des Politiques Sociales ont été ouvertes dans chaque province. Un grand nombre de travailleurs sociaux étaient nécessaires dans ces organisations provinciales. Cependant, comme mentionné ci-dessus, la diffusion irrégulière de la formation en travail social dans tout le pays coïncide également avec les mêmes années. Pour cette raison, les travailleurs sociaux n'ont pas pu être nommés dans de nombreuses organisations provinciales. Des professionnels de différentes professions tels que sociologues, enseignants, pédagogues et infirmières ont été nommés aux postes vacants. Ces nominations suscitent également des critiques de temps en temps.

Aujourd'hui, des dizaines de travailleurs sociaux diplômés du département de travail social en Turquie sont confrontés à un grave problème d'emploi. Le taux de nomination dans des institutions et organisations publiques parmi les diplômés a considérablement diminué par rapport aux 10 années précédentes (Akçay et Alpoğlu, 2020). La période qui a suivi la création du ministère de la Famille et des Politiques Sociales s'est poursuivie jusqu'en 2018. Puis, lors d'un référendum organisé en 2018, la République de Turquie a changé le système parlementaire en "un système présidentiel de gouvernement" et de nombreux ministères ont été fusionnés. Dans ce processus, le ministère de la Famille et des Politiques Sociales a fusionné avec le Ministère du travail et de la sécurité sociale et est devenu le ministère de la Famille, du Travail et des Services Sociaux. Ainsi, les pratiques de travail social rassemblées sous un même toit ont été placées dans un ministère plus complet comprenant différents domaines. Il existe différentes perspectives concernant cette nouvelle réglementation, qui peut être évaluée comme positive ou négative. Le fait que deux ans se soient écoulés depuis ce nouveau règlement 
nous empêche de faire une évaluation objective sur ces sujets. Les développements qui auront lieu dans les années à venir nous permettront de faire une évaluation plus complète du nouveau système.

\section{Conclusion}

Dans cette étude, l'évolution du travail social en Turquie; de la création de la société d'Ankara Himaye-i Etfal en 1921 au ministère de la Famille, du Travail et des Services Sociaux en 2018, a été évaluée en détail depuis la déclaration de république jusqu'à aujourd'hui. La pratique du travail social en Turquie s'est poursuivie après la république dans certaines institutions établies sous l'Empire ottoman. Dans la période suivante, on constate qu'elle a continué son existence en se développant jusqu'au XXIe siècle, en fonction des évolutions nationales et internationales. Dans ce processus, la formation au travail social, qui a commencé en fonction des développements internationaux sous la direction de l'ONU et s'est développée avec des opportunités nationales, est très importante. Donc, le début et la poursuite de la formation en travail social en Turquie jusqu'à présent est un bon exemple dans l'histoire du travail social hybride en Turquie.

Dans le cadre du ministère nouvellement créé en 2018, le travail social s'est appliqué via des aides sociales et des services sociaux pour les familles, les enfants, les femmes, les personnes âgées, les handicapés, les martyrs et les anciens combattants. En outre, des études sont menées sur des questions telles que le droit au travail, le droit à un salaire décent pour un travail décent et une vie professionnelle. Cependant, malgré tout ce travail ou effort, cela ne veut pas dire que le travail social en Turquie ne se réunira probablement pas sous un même toit.

Aujourd'hui, on constate que les pratiques de travail social sont menées dans de nombreuses unités relevant du ministère de la Santé, du ministère de la Justice, du ministère de l'Intérieur, du ministère de l'Éducation Nationale, du ministère de la Jeunesse et des Sports. De plus, la plupart des professionnels travaillant dans ces ministères (tels que les psychologues, les infirmières, les soignants, les sociologues, les enseignants, les animateurs de jeunesse, etc.) ne sont pas des travailleurs sociaux. À ce stade, il est très important que les pratiques de travail social effectuées dans ces unités soient effectuées par des travailleurs sociaux. Ceci n'est pas seulement important pour le bénéfice des clients dans les services offerts, mais aussi pour l'institutionnalisation, l'acceptation sociale et la respectabilité du travail social en Turquie. Comme nous l'avons mentionné ci-dessus, le travail social en Turquie a commencé à être institutionnalisé pendant l'Empire ottoman et ces conditions détermineront à quel point le travail social atteindra au 21e siècle. Espérer un avenir où des pratiques meilleures et plus efficaces seront mises en œuvre par des travailleurs sociaux mieux formés et auto-développés en Turquie.

\section{Conflit d'intérêts}

L'auteur de l'article déclare qu'il n'y a pas de conflit d'intérêts.

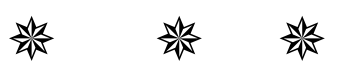




\section{References}

Akçay, S., \& Alpoğlu, İ. E. (2020). Sosyal hizmet uzmanlarının işsizlik deneyimleri. Toplum ve Sosyal Hizmet, 31(1), 47-69.

Alkan, M. N. (2013). Lüksemburg zirvesi krizi. Dans H. Çakmak (Ed.). Türk dış politikasında 41 kriz 19242014, (pp. 251-255). Kripto Yayınevi.

Alptekin, K. (2016). Başlangıçtan bugüne ve yarına Türkiye'de sosyal hizmet eğitimi. Nobel Akademik Yayıncilik.

Alptekïn, K., Topuz, S., \& Zengïn, O. (2017). Türkiye'de sosyal hizmet eğitiminde neler oluyor?. Toplum ve Sosyal Hizmet, 28(2), 50-69.

Altıntaş, Ö. F. (2008). Avrupa Birliği'ne aday ülke olarak Türkiye'de AB uyum yasalarının iç hukuka etki ve katkısı. T.C. Adalet Bakanlığı Yayını.

Artan, T. (2012). Yerel yönetimlerde sosyal hizmetler. SABEV Yayınları.

Association des travailleurs sociaux (2020, Juin 16). Sosyal hizmet uzmanları derneği tüzüğü. http://shuder.org/Sayfa/tuzuk1741

Ateş, E. (2019). 1996- 2002 arası dönemde Türkiye-Avrupa Birliği ilişkilerindeki gelişmeler. Barış Araştırmaları ve Çatışma Çözümleri Dergisi, 7(1), 26-50.

Bay, A. (2017). Osmanlı' da kısa ömürlü bir hayır kurumu: Dulhane (1884-1896). Türkiyat Mecmuasi, 27(1), 47-64.

Bozkurt, R. (2019). Sosyal hizmet uzmanları mesleki örgütlenme süreci ve sosyal hizmet uzmanları derneği. (dans) E. Yeşilkayalı (Ed.). Sosyal çalışma mesleğinde kariyer seçenekleri (pp. 63-80). Nika Yayınları.

Buğra, A. (2016). Kapitalizm, yoksulluk ve türkiye'de sosyal politika. İletişim Yayınları.

Burgaç, M. (2018). Savaşın çocukları: İkinci Dünya Savaşı yıllarında İstanbul'da sokak çocukları sorunu ve çözüm arayışları. Anadolu Üniversitesi Sosyal Bilimler Dergisi, 18(2), 173-182.

Code civil turc (2001, Juin 24). Türk Medeni Kanunu. https://www.mevzuat.gov.tr/MevzuatMetin/1.5.4721.pdf

Conseil de l'enseignement supérieur turc (2020, Juin 3). YÖK Atlas: Üniversitelerimiz. https://yokatlas.yok.gov.tr/universite.php

Constitution turque de 1982 (2020, Mai 19). Mevzuat Bilgi Sistemi. https://www.mevzuat.gov.tr/mevzuat?MevzuatNo=2709\&MevzuatTur=1\&MevzuatTertip=5

Çağlar, D. (1982). Atatürk ve Cumhuriyet Devrinde Korunmaya muhtaç çocuklara sağlanan bakım ve öğretim olanakları. Ankara Üniversitesi Ĕ̆itim Bilimleri Fakültesi Dergisi, 15(2), 1-21.

Çakmak, D. (2007). Toplumsal uzlaşma belgesi: 1936 Tarihli İş Kanunu. Sosyal Bilimler Dergisi, 2007(1), 127-169.

Çavuşoğlu, T. (2001). Türkiye Çocuk Esirgeme Kurumu ve çocuk bayramı. Sosyal Hizmetler Dergisi, 1(1), 9-16. 
Çavuşoğlu, T., \& Çetin, H. (2019). Türkiye'de sosyal hizmetlerin kurumsallaşmasında 1945-1970'li yıllar. (dans) E. Yeşilkayalı (Ed.). Sosyal çalışma mesleğinde kariyer seçenekleri (pp. 23-40). Nika Yayınları.

Çetin, T. (1994). Cumhuriyetin ilk yıllarında köy sorununa bakış: Köy kanununun çıkarılması. Çă̆daş Türkiye Tarihi Araştırmaları Dergisi, 2(4), 29-44.

Duyan V., \& Özgür Bayır, Ö. (2016). Sosyal hizmete giriş: Öğrenciler ve uygulayıcılar için bir rehber. Sosyal Çalışma Yayınları.

Ertem, A. (1999). Osmanlıdan günümüze vakıflar. Divan: Disiplinlerarası Çalı̧̧malar Dergisi, 1(1), 111-150.

Gözen, R. (2006). Türkiye-AB yakınlaşması: 1999 AB Helsinki Zirvesinden 2003 Irak Savaşına stratejik algılamalarda dönüşüm süreci. Uluslararası İlişkiler, 3(10), 115- 146.

Gözler, K. (1999). Türk Anayasaları. Ekin Kitabevi Yayınları.

Gümüş, A. T. (2010). Sosyal devlet anlayışının gelişimi ve dönüşümü (1. Baskı). On İki Levha Yayıncılık.

Güzel, B. (2017). Histoire de la formation au travail social en Turquie. The Journal of Academic Social Science Studies, 56(3), 509-520.

Institution de sécurité sociale (2020, Mai 20). L'histoire du système de sécurité sociale en Turquie. http://www.sgk.gov.tr/wps/portal/sgk/tr/kurumsal/kurumumuz/tarihce

Karaca, C., \& Çam, Y. (2019). Türkiye'de sosyal devlet anlayışının değerlendirilmesi. Maliye Dergisi, 176, $72-97$.

Kanca, O. (2012). 1950-1960 aras1 Türkiye'de uygulanan sosyo-ekonomik politikalar. Mustafa Kemal Üniversitesi Sosyal Bilimler Enstitüsü Dergisi, 9(19), 47-63.

Kesgin, B. (2014). Kamu sosyal politikalarında sosyal yardım. Aç1lım Kitap.

Kesgin, B. (2016). Kamu sorumluluğunda sosyal hizmet. Aç1ım Kitap.

Koçak, D. (2019). 17 Şubat 1926'da kabul edilen Türk Medeni Kanunu'na göre Türk kadınının hak ve özgürlükleri. Atatürk Dergisi, Erzurum Kongresi'nin 100. Yıl Özel Sayısı, 79-98.

Kongar, E. (2007). Sosyal çalışmaya giriş (Tıpkıbasımı). SABEV Yayınevi.

Loi sur la municipalité de 1930 (2020, Mai 15). Resmi Gazete. https://www.resmigazete.gov.tr/arsiv/1471.pdf.

Loi sur la municipalité de 2005 (2020, Mai 27). Belediye Kanunu. https://www.mevzuat.gov.tr/MevzuatMetin/1.5.5393.pdf

Ministère de la Famille, du Travail et des Services Sociaux (2020, Mai 12) Çocuk Hizmetleri Genel Müdürlüğü. https://ailevecalisma.gov.tr/chgm/hakkimizda/tarihce/

Organisation Internationale du Travail (2003, Juin 17). La sécurité sociale pour tous: un pari mondial, Travail Le Magazine de l'OIT, No 49 decembre. https://www.ilo.org/wcmsp5/groups/public/---

Organisation de Planification d'État (2020, Juin 8). Deuxième plan quinquennal de développement (19681972). $\quad$ https://www.sbb.gov.tr/wp-content/uploads/2018/11/\%C4\%B0kinci-Be\%C5\%9FY\%C4\%B111\%C4\%B1k-Kalk\%C4\%B1nma-Plan\%C4\%B1-1968-1972\%E2\%80\%8B.pdf 
Özbek, N. (1999). İkinci Abdülhamid ve kimsesiz çocuklar: Darülhayr-i Ali, Tarih ve Toplum, 31(182), 1120.

Öztürk, A. İ. (2013). Cumhuriyet Döneminde belediyelerin asker aile yardımları. Yakın Dönem Türkiye Araştırmaları, 11(21), 27-57.

Sarıkaya, M., \& Çavuşoğlu, T. (2014). "İstanbul serseri çocuklar projesi” Sosyal hizmet tarihinde kilometre taşları (2. Baskı). Kadın ve Sosyal Hizmetler Vakfı Yayınları.

Savran, S. (2016). Türkiye'de simıf mücadeleleri: Cilt: 1 1908-1980. Yordam Kitap.

Soydan, A. (2003). Darüşşafaka tarihinden kesitler. Yakın Dönem Türkiye Araştırmaları, 3, 249-268.

Şafak, N. (2013). Dârüleytamda çocuk olmak: On çocuk on portre. FSM İlmî Araştırmalar İnsan ve Toplum Bilimleri Dergisi, 2, 261-283.

Şahin Taşğın, N., \& Tekin, U. (2013). Türkiye'de sosyal hizmetin temel karakteristikleri ve güncel görünümü. (dans) H. Acar, N. Nergiz \& E. Akman (Eds.). Sosyal politika ve kamu bileşenleriyle sosyal hizmet temelleri ve uygulama alanları (pp. 119-135). Maya Akademi.

Ünal, V. (2012). Osmanlının son dönemlerinde korunmaya muhtaç çocuklar için kurulan sosyal hizmet kuruluşları. Cumhuriyet Üniversitesi Edebiyat Fakültesi Sosyal Bilimler Dergisi, 36(2), 1-27.

Yıldırım, N. (1997). İstanbul darülaceze müessesesi. Darülaceze Vakfı Yayınları.

Yıldırım, Ş. (2012). Sosyal hizmet biliminin gelişiminde sosyal hizmetler ve Çocuk Esirgeme Kurumu'nun yeri. (dans) İ. C1lga \& B. Hatipoğlu (Eds.). Sosyal bilimler ve sosyal hizmet üzerine düşünceler (pp. 5788). SABEV Yayınları.

Yolcuoğlu, G. (2014). Sosyal hizmet/sosyal çalışma bilim ve mesleğine giriş. Nar Yayınevi.

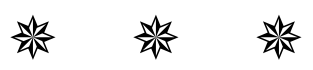


Bekir GÜZEL

\section{ANNEXE}

Photo 1. Enfants sous la protection de la Société Himaye-i Etfal dans les années 1920

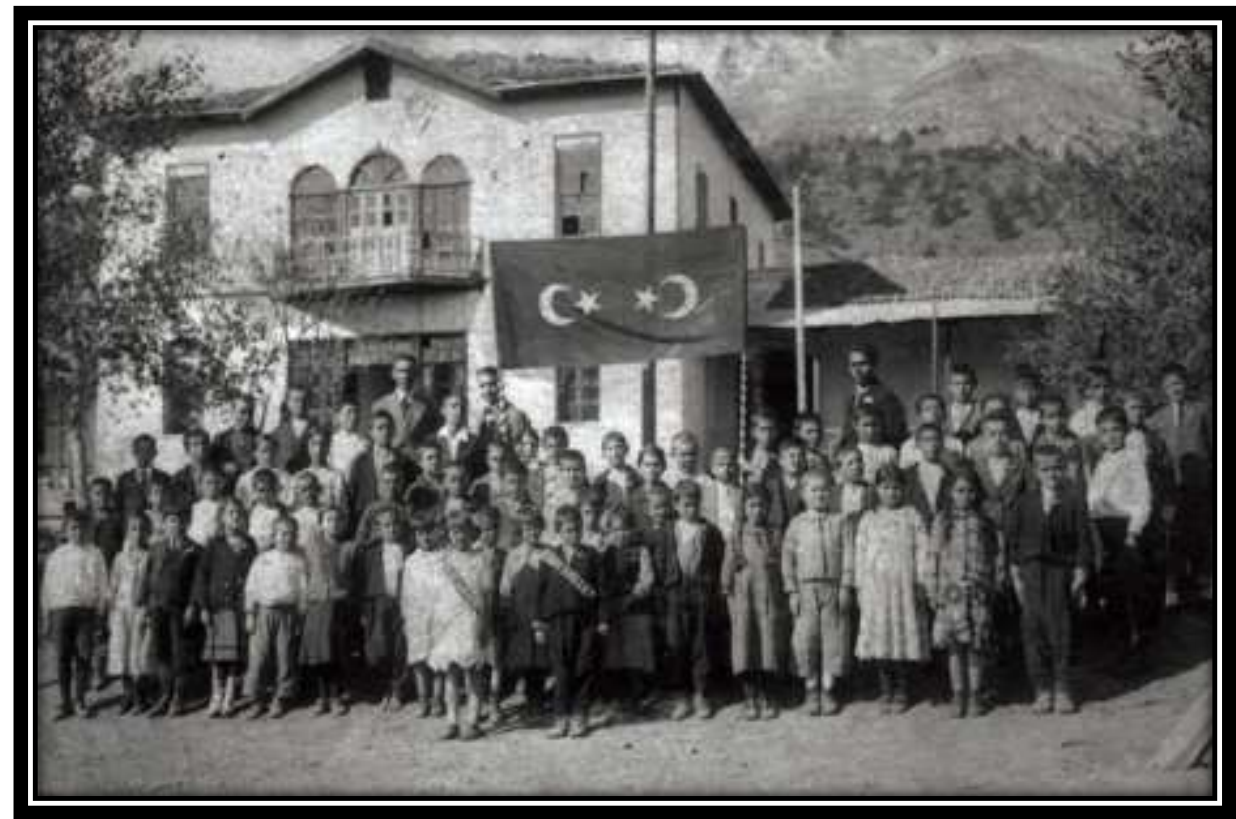

https://listelist.com/gurbuz-cocuk-23-nisan/ (Date d'accès: 28.08.2021)

Photo 2. Lutte contre la tuberculose, Ministère de la Santé et de l'Aide Sociale

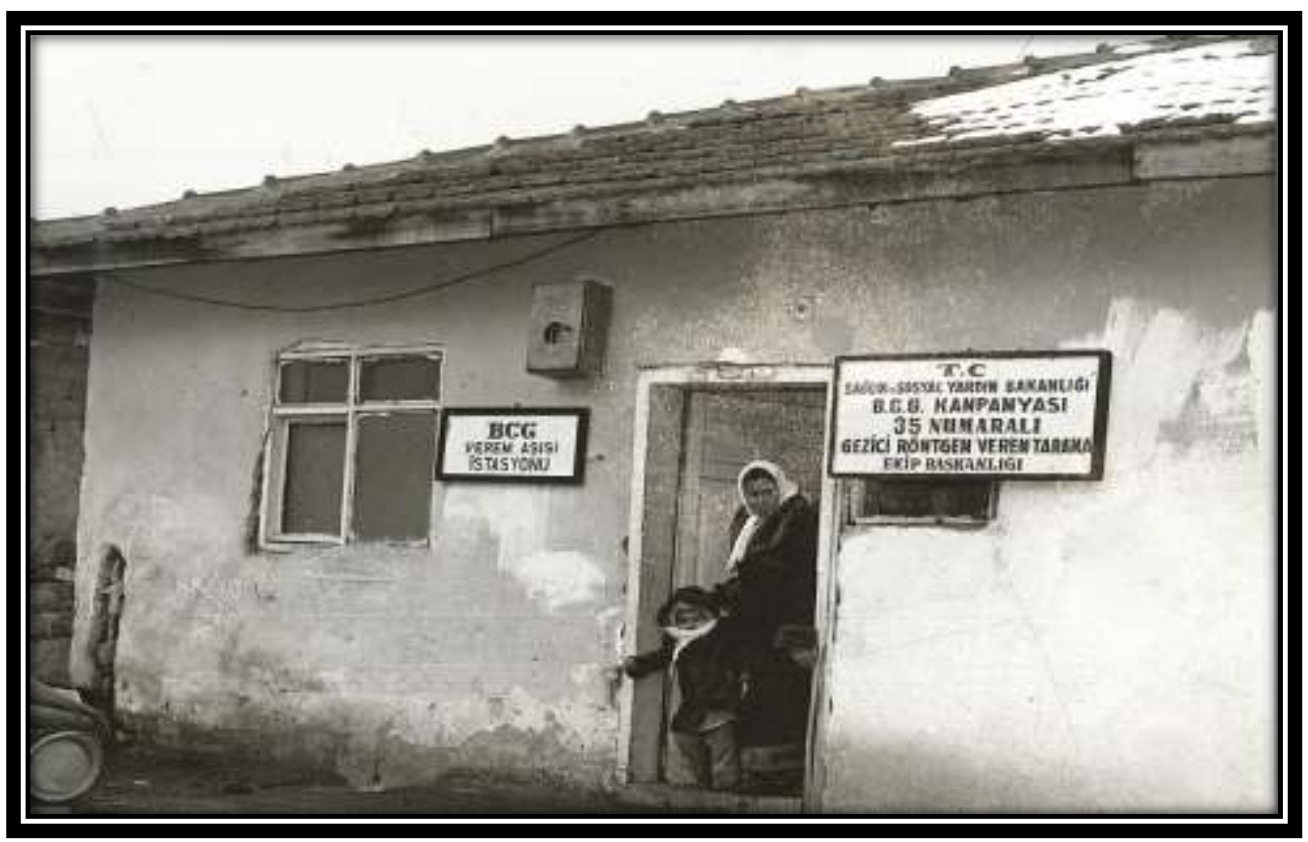

https:/hsgm.saglik.gov.tr/tr/tuberkuloz-baskanligimiz/tarihte.html (Date d'accès: 29.08.2021) 
Photo 3. Le jardin d'enfants d'Atatürk, (Les années 1930 en Ankara)

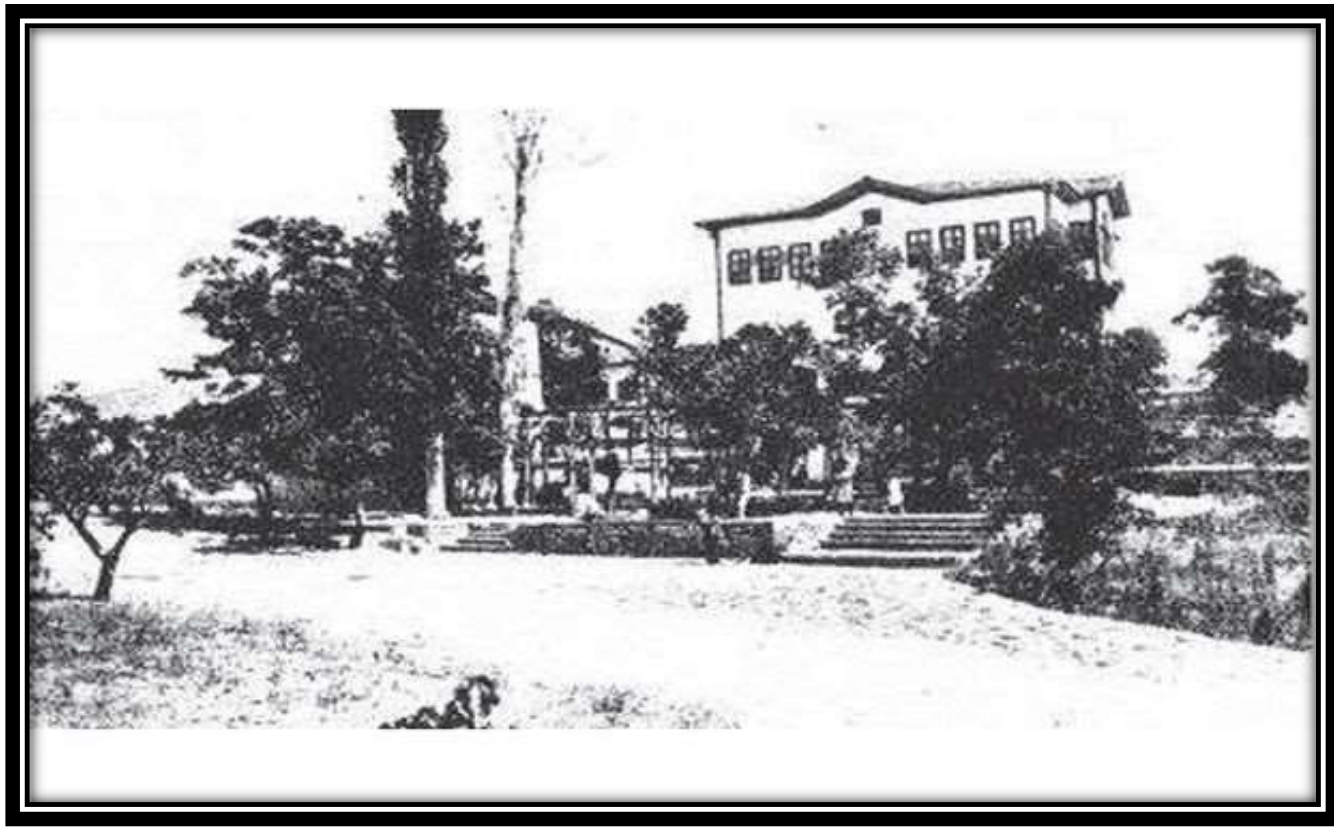

https://www.haberturk.com/gundem/haber/1505439-ataturk-cocuk-yuvasi-muze-olsun-onerisi (Date d'accès: 28.08.2021)

Photo 4. Coupon de loterie de l'Agence de protection de l'enfance, 1951

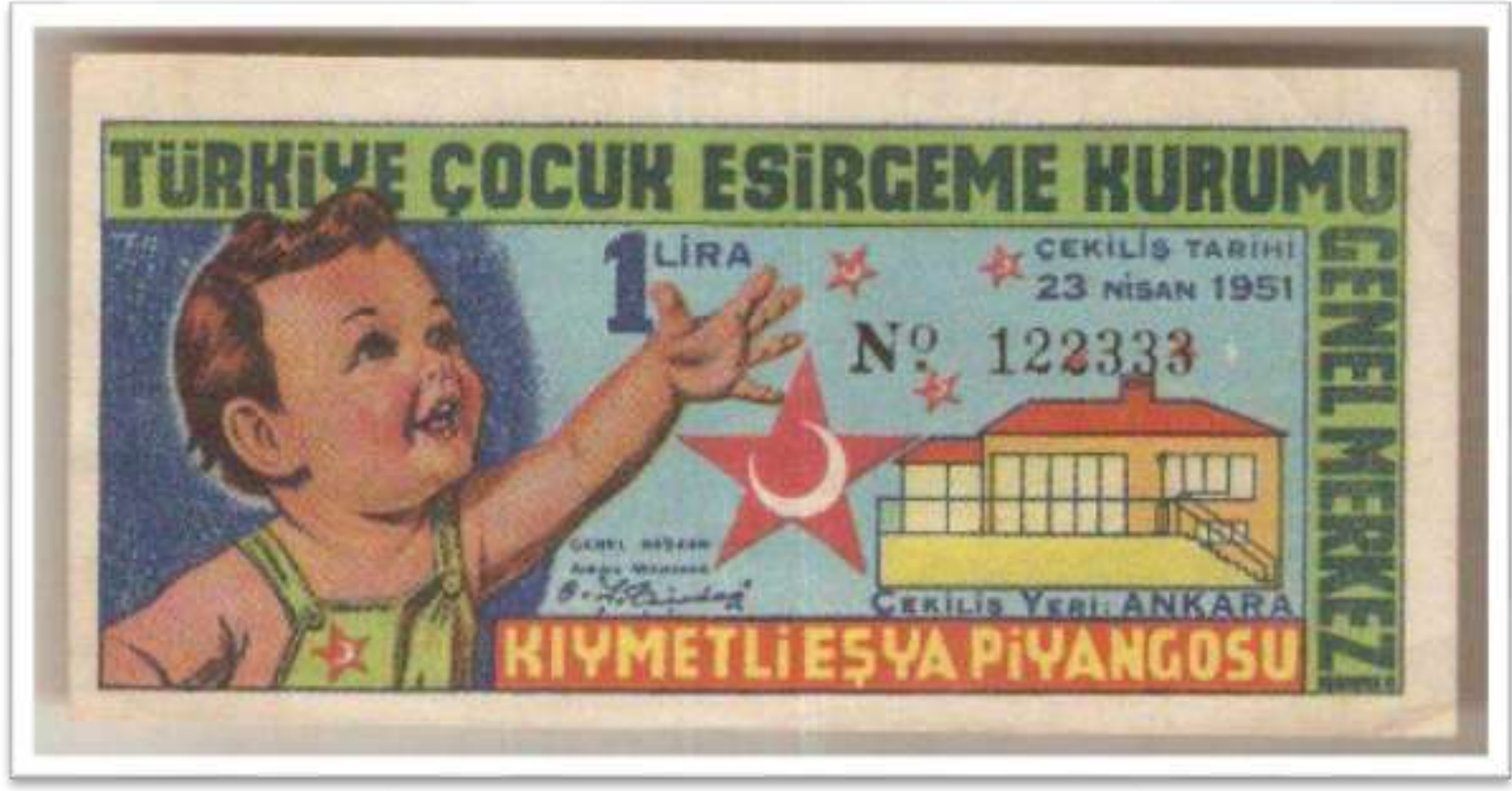

https://www.kitantik.com/product/1951-COCUK-ESIRGEME-KURUMU-ESYAPIYANGOSU_0z8kgltjhj9bmnr1ucl (Date d'accès: 28.08.2021) 
Photo 5. Immeuble Ankara Himaye-i Etfal, appartenant à l'Agence de protection de l'enfance

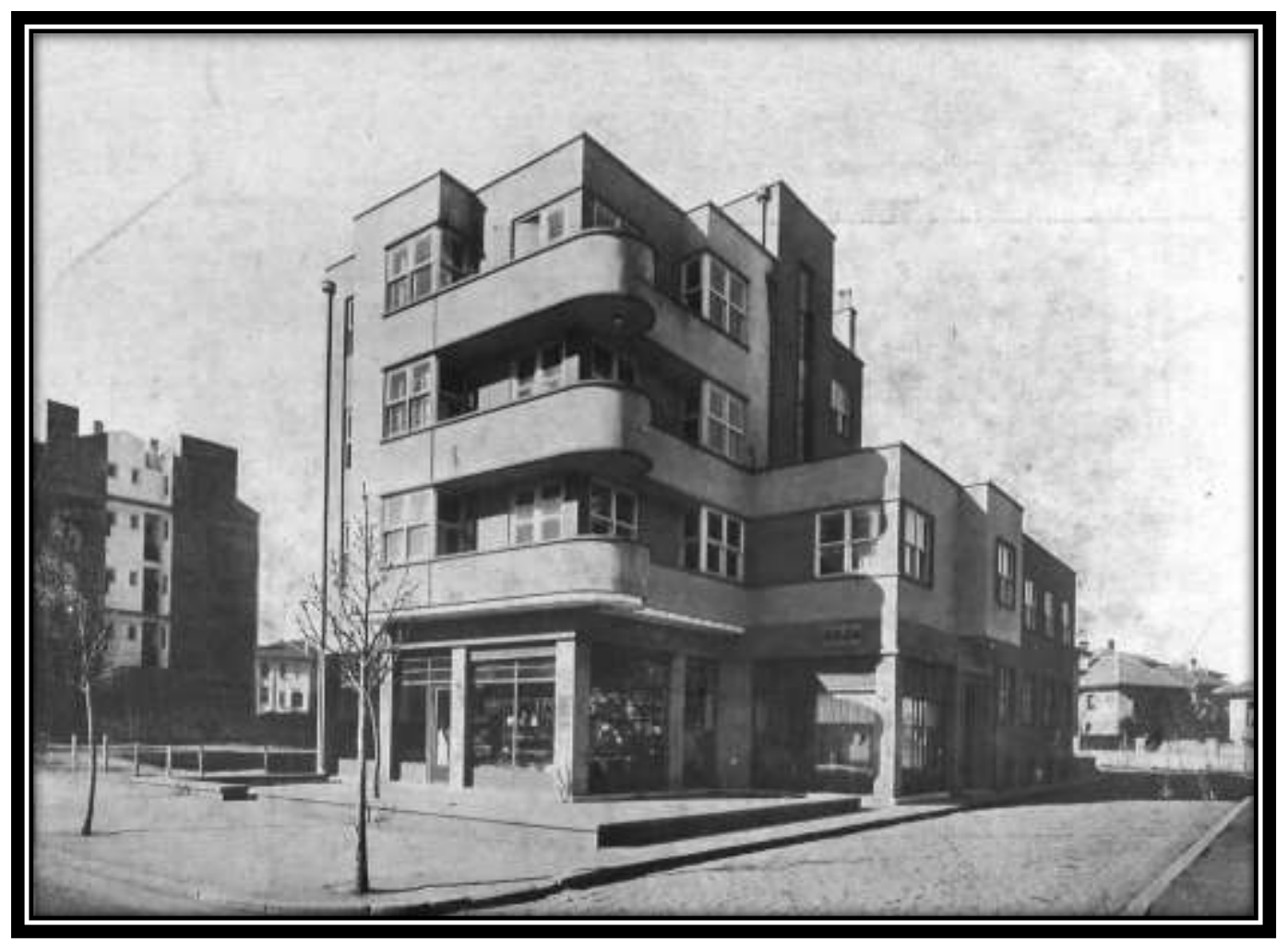

https://odoarchitecture.com/2021/04/13/cocuk-esirgeme-kurumu-ankara-himaye-i-etfal-apartmani/ (Date d'accès: 28.08.2021)

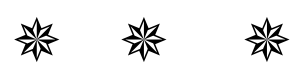

